\title{
Arrico Comparação entre autoimagem e índice de massa corporal entre crianças residentes em favela do Rio de Janeiro, 2012*
}

doi: 10.1590/51679-49742021000100004

\author{
Comparison between self-image and body mass index among children living in a slum \\ in Rio de Janeiro, Brazil, 2012
}

\section{Comparación entre la autoimagen y el índice de masa corporal entre los niños que viven en una favela en Rio de Janeiro, Brasil, 2012}

\author{
Sueli Rosa Gama' - (1) orcid.org/0000-0002-4246-8387 \\ Letícia de Oliveira Cardoso ${ }^{1}$ - (1) orcid.org/0000-0003-1312-1808 \\ Elyne Montenegro Engstrom ${ }^{1}$ - (1) orcid.org/0000-0001-6149-3396 \\ Marilia Sá Carvalho² - (1) orcid.org/0000-0002-9566-0284 \\ 'Fundação Oswaldo Cruz, Escola Nacional de Saúde Pública Sergio Arouca, Rio de Janeiro, RJ, Brasil

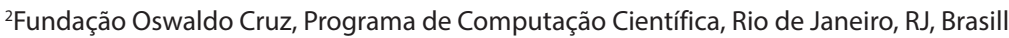

\section{Resumo}

Objetivo: Analisar a concordância/discrepância entre autoimagem corporal e classificação do índice de massa corporal (IMC), segundo tipo de alimentação e influências do ambiente. Métodos: Análise transversal de 195 crianças pré-púberes ( $\geq 5$ anos), atendidas na Atenção Primária à Saúde (APS), Manguinhos, Rio de Janeiro, Brasil. 0 IMC foi classificado conforme escore-z. Avaliou-se a autoimagem pela escala de silhuetas para crianças. Estimou-se a associação entre as covariáveis e subestimação/superestimação do IMC em relação à autoimagem, em modelo multinomial. Resultados: Crianças com sobrepeso subestimaram seu IMC, em comparação com a autoimagem, em maior proporção $(58,6 \%)$ que aquelas com obesidade (22,0\%) ou com eutrofia (49,0\%). Essa dissociação correlacionou-se com a participação no programa de transferência de renda $\left(\mathrm{RC}=2,01-\mathrm{IC}_{95 \%} 1,04 ; 3,90\right)$ e com o consumo diário de alimentos açucarados $\left(\mathrm{RC}=3,88-\mathrm{IC}_{95 \%} 1,05 ; 14,39\right)$. Conclusão: A subestimação do IMC entre as crianças com excesso de peso deve ser considerada pela APS, visando aperfeiçoar as práticas de intervenção.

Palavras-chave: Imagem Corporal; Obesidade Infantil; Índice de Massa Corporal; Atenção Primária de Saúde; Estudos Transversais.

\footnotetext{
*Artigo resultante de tese de doutorado intitulada 'Excesso de peso de crianças moradoras em favela do Rio de Janeiro: uma abordagem sistêmica', defendida por Sueli Rosa Gama junto ao Programa de Epidemiologia em Saúde Pública, da Escola Nacional de Saúde Pública Sergio Arouca (ENSP)/Fundação Instituto Oswaldo Cruz (Fiocruz), Rio de Janeiro, RJ, 2016.

Marilia Sá Carvalho e Elyne Montenegro Engstrom receberam apoio financeiro da Fundação Carlos Chagas Filho de Amparo à Pesquisa do Estado do Rio de Janeiro: E_26/2014-203577; E26/110.280/2012. Marilia Sá Carvalho recebeu apoio do Conselho Nacional de Desenvolvimento Científico e Tecnológico (CNPq)/Ministério da Ciência, Tecnologia, Inovações e Comunicações (MCTIC): PQ 309692/2013.
} 


\section{Introdução}

A prevalência da obesidade infantil vem aumentando, desde 1980, em nível global. Grupos sociais de baixa renda, nos países em desenvolvimento, são especialmente afetados pelo fenômeno., ${ }^{1,2} \mathrm{Na}$ rotina dos serviços da Atenção Primária à Saúde (APS), a principal estratégia de assistência às crianças com excesso de peso é a orientação de suas famílias para escolhas alimentares adequadas, após avaliação nutricional. Entretanto, não é uma tarefa simples. Frequentemente, os pais subestimam 0 índice de massa corporal (IMC) de seus filhos, veem seus corpos grandes como saudáveis, segundo uma percepção culturalmente condicionada. ${ }^{3,-12}$ A imagem corporal das crianças também é um produto da influência familiar, seja porque seus adultos mais próximos apresentam tipos de corpos com os quais a criança se identifica, seja porque estimulam uma autopercepção específica, mediante elogios ou críticas. ${ }^{5}$

\section{A imagem corporal é resultante da aparência física e da representação mental do tamanho e da forma do próprio corpo, nascida de experiências, ações, palavras ou atitudes a ele direcionadas.}

A imagem corporal é resultante da aparência física e da representação mental do tamanho e da forma do próprio corpo, nascida de experiências, ações, palavras ou atitudes a ele direcionadas. Aspectos simbólicos e subjetivos, como afetos, valores e história de vida, também estão presentes na avaliação da imagem corporal. Além disso, onde o excesso de peso é altamente prevalente, a cultura popular tenderá a considerar os valores de IMC acima do recomendado como padrões saudáveis.

A avaliação da consonância/discrepância entre imagem corporal e IMC é essencial, tanto em relação à superestimação, comum entre os adolescentes, ${ }^{6,7}$ quanto à subestimação, na medida em que a imagem corporal e o IMC podem influenciar as ações de promoção do peso saudável. 0 gênero é o principal aspecto da autopercepção, em todos os domínios. ${ }^{8}$ A composição do contexto familiar e cultural está relacionada à avaliação da imagem corporal. A maior escolaridade materna e a maior renda constituem fatores associados a uma percepção da imagem corporal mais próxima do IMC medido.'

Outros fatores devem ser considerados no planejamento de intervenções voltadas às famílias, com 0 propósito de promover a adoção de hábitos alimentares saudáveis. Na maioria dos países em desenvolvimento, as dietas tradicionais foram abandonadas em favor de produtos industrializados, predominantemente ultraprocessados, ${ }^{9}$ em parte devido a uma mudança profunda no papel das mulheres nessas sociedades. Nas regiões mais carentes, é maior a frequência de mulheres provedoras da economia da casa, com pouco tempo disponível para as tarefas domésticas, incluindo cozinhar. ${ }^{8}$ Além do que, o acesso a alimentos industrializados pode simbolizar um processo de ascensão social, melhoria de condições de vida: por exemplo, apesar da oferta de alimentação equilibrada e variada nas escolas públicas brasileiras, o número de crianças que recusam as refeições escolares gratuitas é maior nos grupos com maior renda relativa. ${ }^{10} \mathrm{~A}$ publicidade, direta ou sutil, estimula o desejo por alimentos industrializados e processados, enquanto representação de saúde e alegria, associados a imagens de corpos perfeitos, saudáveis e felizes. ${ }^{11}$

A relação entre os fatores associados ao excesso de peso das crianças e a percepção equivocada de seu status de peso é pouco estudada nos países em desenvolvimento. No Brasil, pesquisas mostram como a cultura corporal valoriza os corpos grandes. ${ }^{12}$ Nas crianças com excesso de peso e que subestimam sua massa corporal, também se verifica maior autoestima. ${ }^{6}$ Estudo realizado na favela de Manguinhos, bairro do município do Rio de Janeiro, no ano de 2012, encontrou um excesso de peso variável entre os sexos/ idades: $36 \%$ em mulheres de 20 a 29 anos; e $55 \%$ em homens de 30 a 59 anos. ${ }^{13}$ Entre as crianças de 5 a 9 anos, da mesma comunidade, $24,8 \%$ situavam-se acima do peso adequado. ${ }^{14}$

Até onde foi possível verificar, não há artigo publicado que avalie a imagem corporal em comunidades de baixa renda, precisamente a população com maior risco de obesidade. Uma melhor compreensão da percepção das crianças poderia contribuir para um melhor atendimento nas unidades básicas de saúde 
(UBS), um espaço positivo para intervenção devido a sua capilaridade e integralidade.

0 objetivo do presente estudo foi analisar a consonância/discrepância entre imagem corporal e classificação do IMC em crianças residentes em favela, segundo tipo de alimentação e influência do ambiente.

\section{Métodos}

Trata-se de análise transversal, de uma coorte aberta de crianças de 5 a 9 anos, iniciada em 2004, que buscava detectar fatores de risco cardiovascular em adultos. Foram estudadas crianças atendidas em duas unidades de APS localizadas em Manguinhos, Rio de Janeiro, RJ. 0 bairro ocupa a $122^{\mathrm{a}}$ posição no índice de desenvolvimento humano (IDH) das 126 regiões do município. A coorte de crianças já foi descrita detalhadamente, em outro artigo. ${ }^{14}$

Primeiramente, o critério de exclusão do estudo foi ser púbere, consultado(a) por autoavaliação, utilizando-se as escalas de Tanner ${ }^{15}$ e com ajuda da pesquisadora. 0 presente consistiu da análise de dados de crianças pré-púberes com 5 anos ou mais de idade, entrevistadas no ano 2012, quando responderam ao questionário de frequência alimentar. Devido à extensão do instrumento, não foi possível aplicá-lo em 158 $(47,5 \%)$ crianças, razão por que acabaram por ser 195 crianças respondentes. Os dados analisados estão disponíveis em arquivo suplementar.
0 IMC foi classificado segundo escore-z da Organização Mundial da Saúde (OMS), ${ }^{16}$ por sexo e idade: baixo peso (escore-z $<-2$ ); peso adequado (escore$-\mathrm{z}>-2$ e $<+1$ ); sobrepeso (escore- $\mathrm{z}>+1$ e $<+2$ ); e obesidade (escore- $z>2$ ). A avaliação antropométrica foi feita na coleta de dados da pesquisa. 0 peso foi medido em balança da marca Filizola, com gradação de $100 \mathrm{~g}$, e as crianças estavam vestidas de roupas leves e sem sapatos. A estatura foi mensurada com fita antropométrica, graduada em $1 \mathrm{~mm}$, presa na parede e a criança sobre ela, com a cabeça ajustada ao plano de Frankfurt.

A imagem corporal foi extraída da escala de silhuetas para crianças, instrumento de pesquisa desenvolvido e avaliado por Kakeshita et al., ${ }^{17}$ composto de 11 formas representativas de corpos de crianças com IMC variando de 12 a $29,85 \mathrm{~kg} / \mathrm{m}^{2}$. Foi utilizada classificação com base nos intervalos do IMC adotados na escala de Kakeshita, e estes valores foram comparados com a referência da Organização Mundial da Saúde 2007 para 0 valor do IMC (Figura 1). Dessa forma, foi possível realizar a associação da autoimagem escolhida com o IMC detectado da criança. 0s cartões com as figuras foram dispostos do menor para o maior tamanho, e as crianças, convidadas a escolher qual imagem consideravam mais próxima de seus próprios corpos.

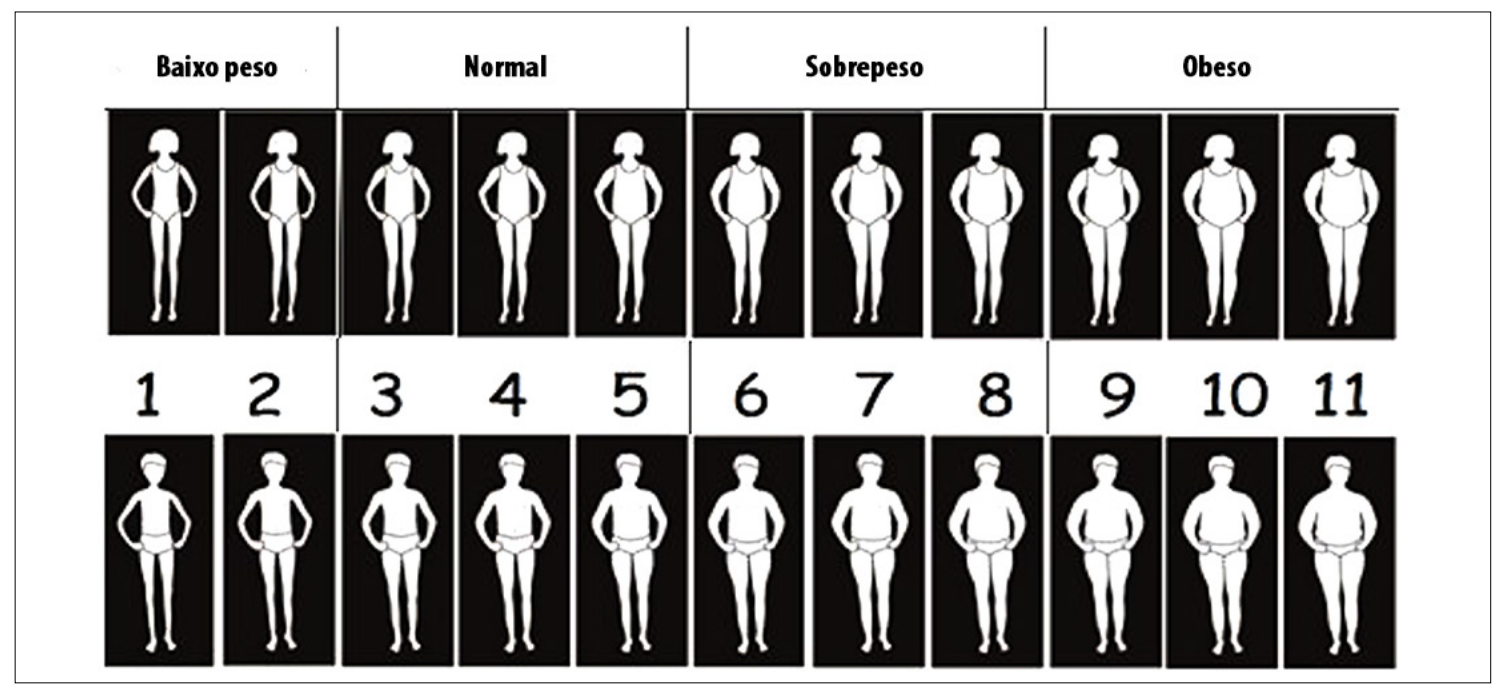

Fonte: Adaptado de Kakeshita et al..$^{17}$

Figura 1 - Escala de silhuetas de crianças com base nos intervalos do índice de massa corporal (IMC) 
Assim, comparou-se a classificação do IMC de cada criança com a classificação da autoimagem corporal, identificando-se superestimação (autoimagem >IMC), subestimação (autoimagem <IMC) e classificação adequada (autoimagem $=$ IMC).

Os fatores potencialmente relacionados ao grau de compatibilidade entre imagem corporal e IMC foram selecionados, e estão descritos a seguir, tendo como referência um modelo teórico desenvolvido em estudo anterior. ${ }^{18}$

A escolaridade da mãe (em anos de estudo: menos de 4; entre 4 e 11; 12 ou mais), indicativo do acesso a informação, que seguiu os níveis usuais do sistema educacional brasileiro, posteriormente consolidados em duas categorias (até 11; 12 ou mais).

A inscrição no programa de transferência de renda intitulado Bolsa Família identifica extrema pobreza. Para descrever a estrutura familiar, foram selecionados: trabalho remunerado da mãe (sim; não); presença do pai na casa (sim; não); presença da avó na casa (sim; não).

Para avaliar o perfil do consumidor, foram utilizados 0 acesso à internet em casa e o consumo da alimentação escolar. Esta variável, inferida pela combinação das informações de frequência e local de cada refeição, indica não apenas um aumento do poder de compra, senão também uma atribuição de valor à capacidade de comprar produtos industrializados. ${ }^{10}$

Os padrões alimentares foram baseados na frequência (diária ou não diária) com que as crianças ingeriam os seguintes alimentos, extraídos do questionário qualitativo de frequência alimentar, validado por Sichieri ${ }^{19}$ e utilizado anteriormente: ${ }^{18}$ carnes industrializadas gordurosas, doces industrializados e bebidas açucaradas, todos considerados produtos ultraprocessados. ${ }^{?}$

Pesquisadores treinados aplicaram um questionário eletrônico aos participantes. A análise descritiva foi estratificada por sexo. Testou-se a associação das variáveis selecionadas com 0 grau de compatibilidade entre a imagem corporal e o IMC, pelo teste do qui-quadrado de Pearson, com nível de significância de 0,10. Este nível de significância é o possível, dado o tamanho da amostra, e aceitável em um trabalho de cunho exploratório. A associação das covariáveis assim selecionadas - com os desfechos 'subestimação' ou 'superestimação' do estado nutricional foi estimada em modelo de regressão logística multinomial, considerando-se a adequação de imagem corporal e IMC como categoria de base. A razão de chances (RC) foi estimada com intervalos de confiança de $95 \%\left(\mathrm{IC}_{95 \%}\right)$. A comparação da adequação entre autoimagem e categorias do IMC (adequação, superestimação e subestimação) foi apresentada graficamente. Utilizou-se 0 software $\mathrm{R}$ para todas as análises estatísticas. ${ }^{20}$

0 projeto do estudo inicial foi aprovado pelo Comitê de Ética em Pesquisa do Instituto Nacional de Saúde da Mulher, da Criança e do Adolescente 'Fernandes Figueira' (CEP/IFF/Fiocruz: Processo $\mathrm{n}^{0}$ 001/04, de 2004) e pelo Comitê de Revisão Ética da Escola Nacional de Saúde Pública Sergio Arouca (CEP/ENSP/ Fiocruz: Processo $n^{0}$ 071/07, de 2007), com Certificado de Apresentação para Apreciação Ética, CAAE $\mathrm{n}^{0}$ 0072.0.031.013-07, emitido em 13 de junho de 2007. Um adendo do projeto, aprovado pelo CEP/ENSP/Fiocruz em 2007, autorizou a participação de crianças no estudo de 2012, após assinatura do Termo de Consentimento Livre e Esclarecido pelos responsáveis.

\section{Resultados}

Foram avaliadas 195 crianças pré-adolescentes, 88 do sexo masculino e 107 do feminino, com idade superior a 5 anos.

A prevalência de crianças com sobrepeso/obesidade foi de $34,9 \%$, proporcionalmente maior entre as meninas. Embora não se detectassem crianças abaixo do peso na amostra, 20,0\% delas avaliavam-se abaixo do peso e 34,3\% consideravam-se acima do peso ou obesas, mais frequentemente no sexo feminino (Tabela 1).

A maior parte das mães tinham entre 4 (apenas alfabetização) e 11 anos de estudo, o que corresponde ao ensino fundamental completo $(57,9 \%)$. Metade das famílias estava inscrita no Programa Bolsa Família (50,3\%). Trabalhavam em emprego remunerado, fora de casa, $47,7 \%$ das mães. Moravam com a mãe $89,2 \%$ das crianças. Em 49,7\% das residências das crianças, o pai estava presente, e em $21,5 \%$, as avós. Não ingeriam as refeições fornecidas na escola $44,1 \%$ das crianças. 0 consumo diário de alimentos doces e bebidas açucaradas foi observado em 70,3\% e 67,7\% das crianças, respectivamente. As carnes gordurosas tradicionais eram consumidas diariamente por apenas $20,5 \%$ das crianças. 
Tabela 1 - Descrição das características da amostra de crianças atendidas ( $n=195)$ em unidade da Atenção Primária à Saúde, Manguinhos, Rio de Janeiro, RJ, 2012

\begin{tabular}{|c|c|c|c|c|c|c|c|}
\hline \multirow{2}{*}{ Variáveis } & \multicolumn{2}{|c|}{ Masculino (88) } & \multicolumn{2}{|c|}{ Feminino (107) } & \multicolumn{2}{|c|}{ Total } & \multirow{2}{*}{$\mathrm{p}$-valor ${ }^{\mathrm{a}}$} \\
\hline & n & $\%$ & n & $\%$ & n & $\%$ & \\
\hline Avaliação nutricional & & & & & & & 0,279 \\
\hline Adequado & 59 & 67,0 & 68 & 63,0 & 127 & 65,1 & \\
\hline Sobrepeso & 15 & 17,0 & 13 & 12,0 & 28 & 14,4 & \\
\hline Obesidade & 14 & 15,9 & 26 & 24,1 & 40 & 20,5 & \\
\hline Imagem corporal & & & & & & & 0,160 \\
\hline Baixo peso & 15 & 17,0 & 24 & 22,2 & 39 & 20,0 & \\
\hline Normal & 48 & 54,5 & 41 & 38,0 & 89 & 45,6 & \\
\hline Sobrepeso & 12 & 13,6 & 21 & 19,4 & 33 & 16,9 & \\
\hline Obesidade & 13 & 14,8 & 21 & 19,4 & 34 & 17,4 & \\
\hline Nível de escolaridade da mãe (anos de estudo) & & & & & & & 0,832 \\
\hline$<4$ & 14 & 15,9 & 19 & 17,6 & 33 & 16,9 & \\
\hline $4-11$ & 53 & 60,2 & 60 & 55,6 & 113 & 57,9 & \\
\hline$\geq 12$ & 20 & 22,7 & 27 & 25,0 & 47 & 24,1 & \\
\hline Inscrição no Programa Bolsa Família & & & & & & & 0,835 \\
\hline Não & 45 & 51,1 & 52 & 49,1 & 97 & 49,7 & \\
\hline Sim & 43 & 48,9 & 55 & 50,9 & 98 & 50,3 & \\
\hline Mãe trabalhando fora de casac & & & & & & & 0,518 \\
\hline Não & 43 & 55,7 & 47 & 50,0 & 90 & 52,3 & \\
\hline Sim & 39 & 44,3 & 54 & 50,0 & 93 & 47,7 & \\
\hline Refeição na escola & & & & & & & 0,285 \\
\hline Não & 43 & 48,9 & 43 & 40,2 & 86 & 44,1 & \\
\hline Sim & 45 & 51,1 & 64 & 59,8 & 109 & 55,9 & \\
\hline Mora com a mãe & & & & & & & 0,635 \\
\hline Sim & 77 & 87,5 & 97 & 89,8 & 174 & 89,2 & \\
\hline Não & 11 & 11,5 & 10 & 9,3 & 21 & 10,8 & \\
\hline Mora com o paid & & & & & & & 0,346 \\
\hline Sim & 40 & 45,5 & 57 & 53,3 & 97 & 49,7 & \\
\hline Não & 48 & 54,5 & 50 & 46,7 & 98 & 50,3 & \\
\hline Mora com a avód & & & & & & & 0,214 \\
\hline Sim & 23 & 26,1 & 19 & 17,6 & 42 & 21,5 & \\
\hline Não & 65 & 73,9 & 88 & 88,2 & 153 & 78,5 & \\
\hline Consumo de carnes gordurosas & & & & & & & 0,066 \\
\hline Não diariamente & 68 & 77,3 & 87 & 87,3 & 155 & 79,5 & \\
\hline Diariamente & 20 & 22,7 & 20 & 18,7 & 40 & 20,5 & \\
\hline Consumo de doces & & & & & & & 0,918 \\
\hline Não diariamente & 27 & 30,7 & 31 & 29,0 & 58 & 29,7 & \\
\hline Diariamente & 61 & 69,3 & 76 & 71,0 & 137 & 70,3 & \\
\hline
\end{tabular}


Continuação

Tabela 1 - Descrição das características da amostra de crianças atendidas ( $n=195)$ em unidade da Atenção Primária à Saúde, Manguinhos, Rio de Janeiro, RJ, 2012

\begin{tabular}{|c|c|c|c|c|c|c|c|}
\hline \multirow{2}{*}{ Variáveis } & \multicolumn{2}{|c|}{ Masculino (88) } & \multicolumn{2}{|c|}{ Feminino (107) } & \multicolumn{2}{|c|}{ Total } & \multirow{2}{*}{ p-valor } \\
\hline & $\mathbf{n}$ & $\%$ & $\mathbf{n}$ & $\%$ & $\mathbf{n}$ & $\%$ & \\
\hline Consumo de bebidas açucaradas & & & & & & & 1,000 \\
\hline Não diariamente & 28 & 31,8 & 35 & 32,7 & 63 & 32,3 & \\
\hline Diariamente & 60 & 68,2 & 72 & 67,3 & 132 & 67,7 & \\
\hline
\end{tabular}

Notas: a) Teste do qui-quadrado de Pearson; b) Duas informações faltantes; c) 12 informações faltantes; d) A presença de cada familiar contada separadamente, para cada criança.

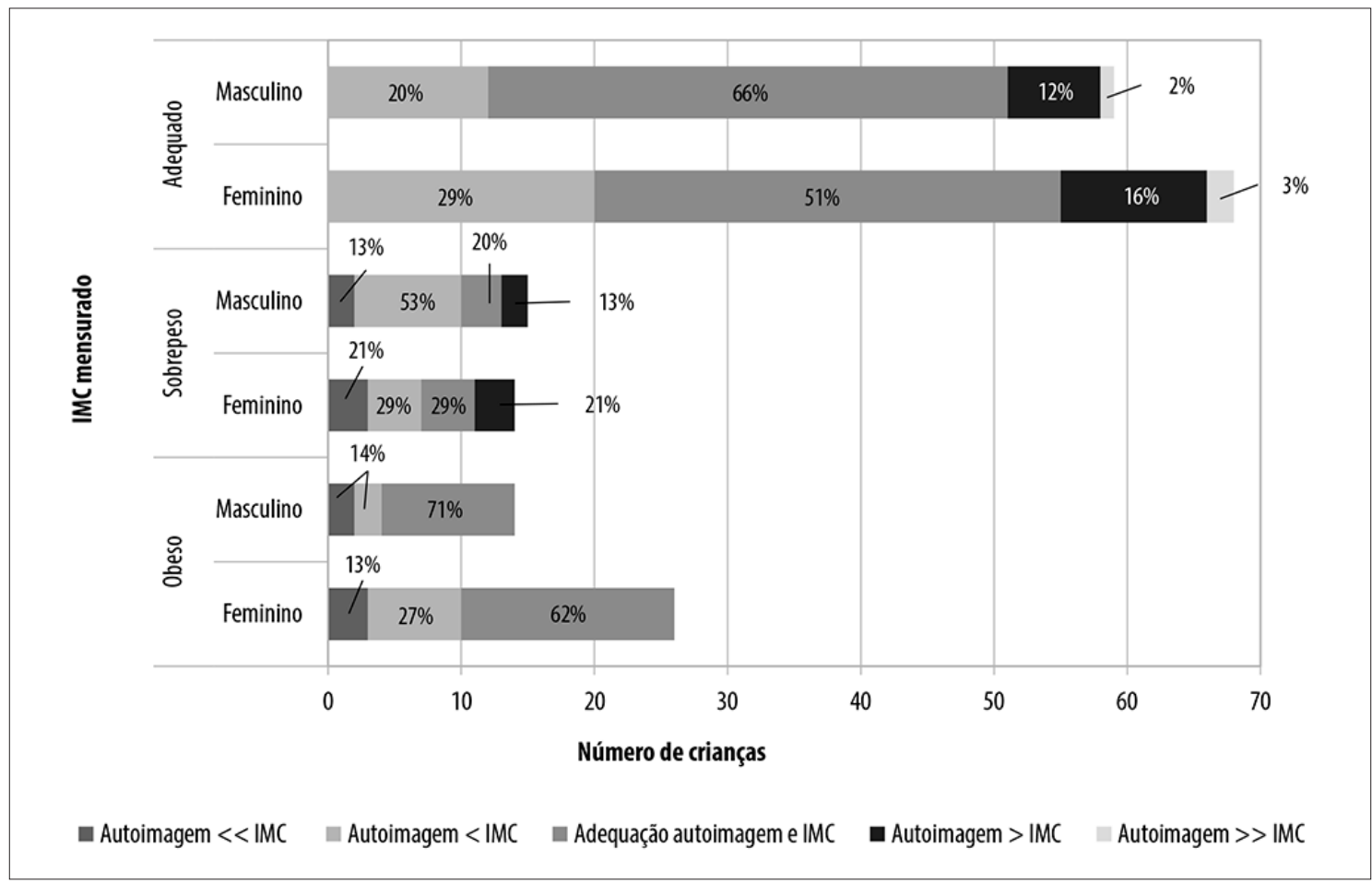

Nota: a) IMC: índice de massa corporal.

\section{Figura 2 - Comparação entre representação da imagem corporal e IMC, a de acordo com 0 sexo}

A Figura 2 apresenta a concordância/discrepância entre imagem corporal e IMC, segundo categorias da classificação do IMC. Em cada uma destas, quando comparados meninos e meninas, o comportamento mostrou-se similar, ainda que as proporções de adequação da imagem corporal fossem sempre maiores entre os meninos.

A discrepância entre imagem corporal e avaliação nutricional foi maior no grupo classificado como de sobrepeso. Entre as crianças obesas, a classificação da imagem corporal foi mais próxima de seu próprio IMC, para ambos os sexos. Mais crianças classificadas no grupo IMC eutrófico avaliaram-se abaixo do peso do que com excesso de peso.

Apenas três variáveis foram associadas a discrepância entre autoimagem corporal e IMC, sempre no sentido da subestimação (Figura 3): estar inscrito no Programa Bolsa Família (p-valor $=0,067)$, consumo diário de doces industrializados ( $\mathrm{p}$-valor $=0,064$ ) e de bebidas açucaradas ( $\mathrm{p}$-valor $=0,094$ ). 


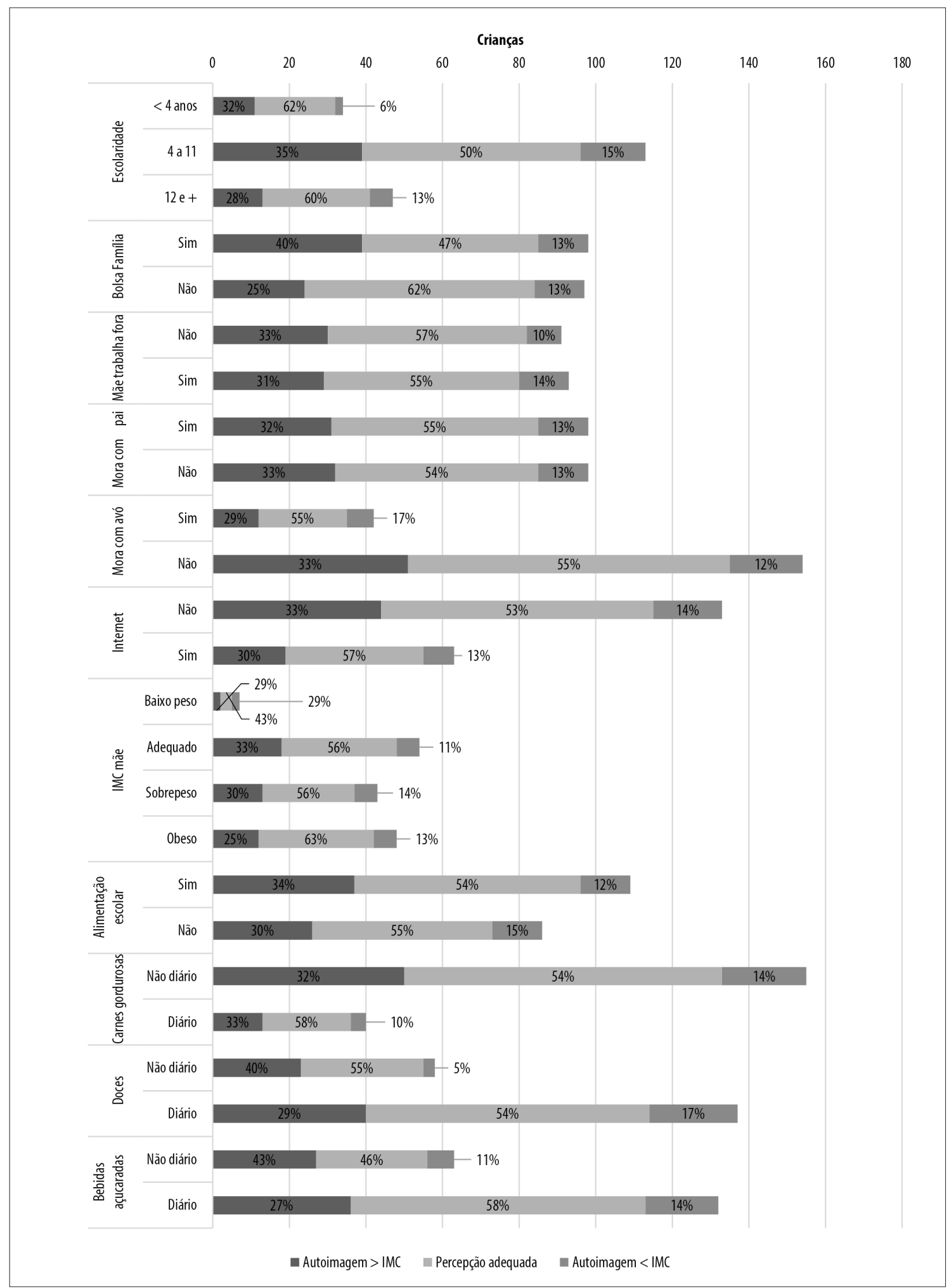

Nota: a) IMC: índice de massa corporal.

Figura 3 - Associação da concordância/discrepância entre autoimagem corporal, IMCa e variáveis selecionadas, de crianças atendidas ( $\mathrm{n}=195$ ) em unidade da Atenção Primária à Saúde de Manguinhos, Rio de Janeiro, RJ, 2012 
Tabela 2 - Associação entre superestimação ou subestimação da classificação nutricional e variáveis selecionadas entre crianças atendidas ( $n=195$ ) em unidade da Atenção Primária à Saúde, Manguinhos, Rio de Janeiro, RJ, 2012

\begin{tabular}{|c|c|c|c|c|c|c|}
\hline \multirow[t]{2}{*}{ Categorias de respostas variáveis } & \multicolumn{3}{|c|}{$\begin{array}{l}\text { Superestimação versus } \\
\text { classificação adequada }\end{array}$} & \multicolumn{3}{|c|}{$\begin{array}{l}\text { Subestimação versus } \\
\text { classificação adequada }\end{array}$} \\
\hline & $\mathbf{n}$ & $\mathbf{R C}^{\mathbf{a}}$ & IC $_{95 \%}^{b}$ & $\mathbf{n}$ & $\mathbf{R C}^{\mathbf{a}}$ & IC $_{95 \%}{ }^{b}$ \\
\hline Inscrição no Bolsa Família (Sim) & 13 & 1,60 & 0,$67 ; 3,80$ & 39 & 2,01 & 1,$04 ; 3,90$ \\
\hline Consumo de doces (diariamente) & 23 & 3,88 & 1,$05 ; 14,39$ & 40 & 1,02 & 0,$50 ; 2,07$ \\
\hline Consumo de bebidas açucaradas & 19 & 0,88 & 0,$33 ; 2,39$ & 36 & 0,54 & 0,$27 ; 1,06$ \\
\hline
\end{tabular}

Notas: a) RC: razão de chances; b) IC $\mathrm{I}_{95 \%}$ : intervalo de confiança de $95 \%$.

A subestimação do IMC foi mais frequente em crianças cujas famílias estavam inscritas no Bolsa Família. Crianças cujas mães tinham menor nível educacional foram as que mais subestimaram seu IMC, diferença não estatisticamente significativa. No entanto, quando as duas categorias de menor escolaridade foram agregadas, o grupo com ensino médio/superior apresentou maior proporção de autopercepção adequada. A discrepância entre imagem corporal e IMC foi um pouco mais frequente entre as crianças que comiam refeições na escola, embora essa associação não fosse significativa (Figura 3).

Mais da metade das mães trabalhava fora de casa. A situação de trabalho das mães não apresentou diferença quanto à concordância/discrepância entre autopercepção do corpo e IMC. Também não houve diferença para a presença do pai ou da avó em casa, como também para o uso da internet.

0 consumo de alimentos ultraprocessados foi frequente nas crianças avaliadas; e das que não consumiam esses produtos todos os dias, uma parcela substancial (aproximadamente 40,0\%) subestimava a classificação do IMC. Não se observou associação da concordância/discrepância entre a imagem corporal e IMC, com o consumo de alimentos gordurosos mais tradicionais, como linguiças, mortadelas, torresmo etc. (Figura 3).

A Tabela 2 apresenta os resultados da regressão logística multinomial. As crianças cujas famílias estavam inscritas no Bolsa Família, frente às não matriculadas no programa, apresentaram duas vezes mais chances de subestimar seu IMC, quando comparadas pela adequação entre autoimagem e IMC $\left(\mathrm{RC}=2,01-\mathrm{IC}_{95 \%}\right.$ $1,04 ; 3,90)$. Por sua vez, uma superestimação do IMC foi observada entre crianças que comem doces todos os dias, quase quatro vezes maior $\left(\mathrm{RC}=3,88-\mathrm{IC}_{95 \%}\right.$
$1,05 ; 14,39)$ quando comparadas com crianças que consomem esses alimentos menos frequentemente.

\section{Discussão}

Neste estudo, realizado sobre dados de crianças residentes em uma comunidade de baixa renda, encontrou-se uma dissociação entre imagem corporal - avaliação subjetiva - e classificação do estado nutricional com base no IMC - medida objetiva.

A maioria dos estudos avalia a imagem corporal das crianças pelo cuidador ${ }^{5}$ bem como a imagem que a mãe percebe da criança; e apenas alguns avaliam as crianças nessa faixa etária. Segundo este estudo, as crianças com sobrepeso subestimaram seu peso com mais frequência do que as crianças obesas, em contraste com outras pesquisas, nas quais crianças obesas subestimaram com mais frequência seu IMC. Ademais, o sexo não esteve associado à concordância/ discrepância entre imagem corporal e IMC, outra diferença em relação ao observado em outros trabalhos, que encontraram associação de discrepância/concordância com o sexo. ${ }^{7,21}$

Vale lembrar que as silhuetas da escala utilizada se baseiam em imagens corporais, construídas sobre os valores de IMC, foram validadas no Brasil e apresentam alta correlação com esse indicador. ${ }^{17}$ Um aspecto relevante chamou a atenção: a habilidade cognitiva da criança em identificar seu tamanho corporal com 0 tamanho corporal ideal, nas idades de 3 a 5 anos. Estudos com mães afro-americanas mostram preferência pelo excesso de peso em seus filhos, valorizando corpos grandes e subestimando 0 grau de excesso de peso. ${ }^{22}$ Indivíduos cujos pais ou colegas apresentam sobrepeso têm maior probabilidade de se perceberem com baixo peso $^{21}$ e uma grande proporção de mães subestima 0 excesso de peso de seus filhos. ${ }^{4}$ 
Outros estudos encontraram associação entre menor posição socioeconômica e subestimação de peso.,22 Como a população avaliada é predominantemente pobre, a principal variável que permite a diferenciação do status socioeconômico é receber o subsídio do programa de transferência de renda, o Bolsa Família, um indicativo de extrema pobreza. No Brasil, os indivíduos podem se qualificar para esse programa se sua renda familiar per capita estiver abaixo de US\$1,78/ dia (valor estabelecido no momento da realização do trabalho de campo do estudo inicial). A partir de 2002, programas sociais relevantes foram implementados no país, contribuindo para o aumento do poder de compra das famílias, o que levou ao surgimento de um grupo social cujas representações corporais, todavia, devem ser estudadas. ${ }^{23}$

0 estado nutricional reflete a melhoria das condições de vida da população: ${ }^{24}$ a desnutrição infantil na região Sudeste, de 13,4\% em 1975, decresceu para apenas 4,1\% em 1989. ${ }^{25}$ Em Manguinhos, 2,0\% estavam desnutridos e $21,7 \%$ acima do peso, ${ }^{14} \mathrm{e}$, nesse contexto, é possível que um corpo magro seja associado a alimentação insuficiente e/ou problemas de saúde.

0 nível de escolaridade da mãe, outro indicador socioeconômico, não se associou significativamente à concordância/discrepância entre percepção subjetiva e IMC, embora os maiores níveis de escolaridade tenham apresentado maior concordância, achado semelhante ao de outros estudos. ${ }^{5,6}$ Entretanto, na China, pesquisa de alcance nacional, realizada em 2010, entre crianças nas escolas primárias, observou que meninos com excesso de peso, de mães com maior nível escolar, apresentaram maior chance de autoimagem adequada. ${ }^{7}$

Na cultura popular, a imagem corporal não segue os padrões de beleza comumente retratados pelos meios de comunicação de massa, quais sejam, normalmente de homens e mulheres altos, magros e brancos, não representativos da presença física hegemônica na realidade local. Infelizmente, não se analisaram as características do cuidador nesta amostra, pois as informações sobre o IMC da mãe apresentavam muitos dados ausentes. A variável raça/cor da pele foi incluída na primeira e na segunda ondas do estudo, ${ }^{14} \mathrm{e}$ como não estava relacionada a qualquer outro indicador socioeconômico ou nutricional, tampouco foi incluída nesse seguimento. Além disso, como a imagem corporal seria solicitada às crianças, à raça também caberia sê-lo, porém inexiste avaliação dessa questão nessa faixa etária.

Igualmente não se observou significância estatística para algumas variáveis relacionadas ao ambiente familiar, principalmente a presença da avó no domicílio, o que poderia representar um legado de hábitos alimentares tradicionais e, possivelmente, uma percepção de que os corpos magros não são saudáveis, estimulando 0 excesso de peso. ${ }^{26}$ Contudo, as avós costumam ser bem jovens nessa comunidade, onde a maternidade é mais ocorrente na adolescência. ${ }^{27}$ Outrossim, como 0 presente estudo foi realizado em um único bairro, não foi possível avaliar o contexto ambiental.

A estrutura familiar, na amostra investigada, não contava com a presença do pai em metade das residências, apontando para um modelo familiar não nuclear, razão por que não se pôde verificar sua associação com a percepção inadequada entre autoimagem e IMC. Entretanto, a participação das mulheres no mercado de trabalho continua a crescer nesse grupo socioeconômico, empregadas em serviços de manutenção e limpeza, trazendo duas questões complementares: 0 aumento da renda familiar, quando a mulher trabalha fora, e a redução em seu tempo disponível para as tarefas domésticas. ${ }^{8}$ Consequentemente, reduz-se o hábito de cozinhar em casa, substituído por preparações prontas.

Outra questão importante foi a redução no consumo de refeições escolares nessa comunidade, demonstrada em estudo anterior, que constatou um decréscimo do hábito, de 73,3\% em 2004 para 48,7\% em 2012. ${ }^{24}$ Entre as crianças que subestimaram seu IMC, encontrou-se menor consumo de refeições escolares. No entanto, comer refeições escolares é tradicionalmente associado à extrema pobreza, ${ }^{10} \mathrm{e}$ um aumento no poder de compra pode explicar sua redução. A substituição de refeições escolares por lanches prontos, principalmente produtos ultraprocessados, valorizados pela publicidade direcionada às crianças, pode ser um marcador de atribuição social de valor, ${ }^{28}$ além do que, crianças com percepção inadequada de peso podem apresentar comportamento alimentar pouco saudável, como 0 alto consumo de doces e salgadinhos. ${ }^{29}$

A compreensão da dissociação entre imagem corporal e IMC transcende o campo das medições e diagnósticos biológicos, devendo-se, portanto, considerar dimensões mais subjetivas das percepções corporais que cada criança constrói, com base em 
seu relacionamento com a família e a sociedade que a cerca. Essas ideias são estudadas com mais profundidade nas Ciências Sociais, por autores como Pierre Félix Bourdieu (1930-2002), para quem as relações entre os corpos físico e social são mediadas pelos capitais econômico, cultural e social, o 'capital simbólico' do indivíduo em sua relação com o mundo. Para analisar esses fenômenos, tanto a formulação de modelos complexos quanto a adoção de metodologias de pesquisa que agregam diferentes campos do conhecimento, como a Epidemiologia e as Ciências Sociais, são estratégias complementares e potentes. Este trabalho apresenta uma análise exploratória sobre essas questões, e suas interpretações beneficiaram-se, sobretudo, de abordagens qualitativas de um estudo que capturou, com maior profundidade, os significados e símbolos envolvidos na imagem corporal. ${ }^{30}$

Apesar do pequeno tamanho amostral de crianças, no modelo multinomial múltiplo, duas variáveis associaram-se significativamente a algum tipo de discrepância: ser atendida pelo Programa Bolsa Família; e comer doces diariamente. As crianças beneficiárias do Programa Bolsa Família apresentaram duas vezes mais chances de subestimar o IMC. Este resultado corrobora os de outros estudos, segundo os quais famílias inscritas no programa, justamente as mais pobres, apresentam corpos grandes, levando suas crianças a perceberem o próprio corpo menor que o real, independentemente de serem classificadas com obesidade, sobrepeso ou eutrofia. A subestimação de seu IMC, por essas crianças, é relevante para os profissionais da APS, devido ao crescente número de pessoas com sobrepeso no país. ${ }^{1,2}$ Entretanto, as crianças que comem balas todos os dias avaliaram-se maiores que a medida objetiva do IMC.

Não obstante a limitação, reconhecida, do pequeno tamanho da amostra, trata-se do primeiro estudo no
Brasil a abordar a discrepância entre a imagem corporal e uma medida objetiva de crianças que vivem em bairros de baixa renda, onde a obesidade infantil é um problema crescente. ${ }^{3}$

A subestimação do IMC em crianças com excesso de peso é preocupante, podendo indicar uma autoimagem com impactos futuros, no ganho de peso. A compreensão dos fatores associados à imagem corporal é essencial para a elaboração tipos de intervenção adequados ao contexto, com o objetivo de alcançar uma alimentação e peso saudáveis. Intervenções sobre excesso de peso exclusivamente normativas, sem se considerar a percepção dos indivíduos, são ineficazes. Outras questões, principalmente a insatisfação corporal entre crianças e adolescentes, também devem ser mais bem estudadas em populações de baixa renda.

Atualmente, esse grupo populacional está passando por profundas mudanças sociais, entre as quais 0 acesso massivo a alimentos ultraprocessados, a redução no tempo de preparação de refeições e a adoção de estilos de vida representativos de maior status social, culturalmente valorizados. É necessário desenvolver novas abordagens na Atenção Primária à Saúde, com respeito à autonomia, capacitação para escolhas saudáveis, autocuidado, e desenvolvimento de práticas educacionais participativas, menos autoritárias ou impositivas.

\section{Contribuição das autoras}

Gama SR e Cardoso L0 contribuíram na concepção e delineamento do artigo, análise e interpretação dos dados e redação da primeira versão do manuscrito. Engstrom EM e Carvalho MS contribuíram na análise e interpretação dos dados e revisão crítica do manuscrito. Todas as autoras aprovaram a versão final e são responsáveis por todos os aspectos do trabalho, incluindo a garantia de sua precisão e integridade.

\section{Referências}

1. Chooi YC, Ding C, Magkos F. The epidemiology of obesity. Metabolism [Internet]. 2019 Mar [cited 2020 Feb 1];92:6-10. Available from: https:// doi.org/10.1016/j.metabol.2018.09.005

2. Wang Y, Lim H. The global childhood obesity epidemic and the associationbetween socio-economic status and childhood obesity. Int Rev Psychiatry [Internet].
2012 Jun [cited 2020 Sep 9];24 (3):176-88. Available

from: https://doi.org/10.3109/09540261.2012.688195

3. Ling J, Stommel M. Parental and self-weight perceptions in U.S. children and adolescents, NHANES 2005-2014. West J Nurs Res [Internet]. 2018 Jan [cited 2019 0ct 10];41(1):42-57. Available from: https://doi.org/10.1177/0193945918758274 
4. Pedroso J, Toral N, Gubert MB. Maternal perception of children's nutritional status in the Federal District, Brazil. PLoS 0ne [Internet]. 2017 Apr [cited 2018 Nov 24]; 12(4):e 0176344. Available from: https://doi.org/10.1371/journal.pone.0176344

5. Tang A, Ji M, Zhang Y, Zou J, Li M, Yang L, et al. Dietary behaviors and caregiver perceptions of overweight and obesity among chinese preschool children. Int J Environ Res Public Health [Internet]. 2018 Apr [cited 2019 0ct 10];15(4):716. Available from: https://dx.doi.org/10.3390\%2Fijerph15040716

6. Jankauskiene R, Baceviciene M. Body image concerns and body weight overestimation do not promote healthy behaviour: evidence from adolescents in Lithuania. Int J Environ Res Public Health [Internet]. 2019 Mar [cited 2019 0ct 10];16(5):864. Available from: https://dx.doi.org/10.3390\%2Fijerph16050864

7. Zhang J, Zhai Y, Feng XQ, Li WR, Lyu YB, Astell-Burt T, et al. Gender differences in the prevalence of overweight and obesity, associated behaviors, and weightrelated perceptions in a national survey of primary school children in China. Biomed Environ Sci [Internet]. 2018 Jan [cited 2020 Sep 9];31(1):1-11. Available from: https://doi.org/10.3967/bes2018.001

8. Sorj B. Arenas de cuidado nas interseções entre gênero e classe social no Brasil. Cad Pesqui [Internet]. 2013 maio-ago [citado 2019 out 12];43(140):478-91. Disponível em: https://doi. org/10.1590/S0100-15742013000200006

9. Monteiro CA, Moubarac J-C, Cannon G, Ng SW, Popkin B. Ultra-processed products are becoming dominant in the global food system: ultra-processed products: global dominance. Obes Rev [Internet]. 2013 Nov [cited 2019 Sep 17];14(Suppl 2):21-8. Available from: https://doi.org/10.1111/obr.12107

10. Hoffmann R. Determinantes do consumo da merenda escolar no Brasil: análise dos dados da PNAD de 2004 e 2006. Segur Aliment Nutr [Internet]. 2012 [citado 2020 set 9];19(1):33-45. Disponível em: https://doi.org/10.20396/san.v19i1.8634667

11. Sadeghirad B, Duhaney T, Motaghipisheh S, Campbell NRC, Johnston BC. Influence of unhealthy food and beverage marketing on children's dietary intake and preference: a systematic review and meta-analysis of randomized trials: Meta-analysis of unhealthy food and beverage marketing. Obes Rev [Internet]. 2016 0ct [cited 2019 0ct 12];17(10):945-59. Available from: https://doi.org/10.1111/obr.12445
12. Goldenberg M. The body as capital: understanding Brazilian culture. Vibrant [Internet]. 2010 Jan-Jun [cited 2020 Sep 9];7(1):220-38. Available from: http://www.vibrant.org.br/issues/ v7n1/mirian-goldenberg-the-body-as-capital/

13. Carvalho MS, Mello AC, Rabello RS. Como está sua saúde? Resultados da pesquisa sobre a situação de saúde dos moradores da região de Manguinhos [Internet]. Rio de Janeiro: Fundação Oswaldo Cruz; 2015 [citado 2015 nov 6]. 12 p. Disponível em: http://www.icict. fiocruz.br/sites/www.icict.fiocruz.br/files/Saude $\% 20$ Urbana_Projeto\%20Teias_boletim_corrigido.pdf

14. Gama SR, Carvalho MS, Cardoso LO, Chaves CRMM, Engstrom EM. Cohort study for monitoring cardiovascular risk factors in children using a primary health care service: methods and initial results. Cad Saúde Pública [Internet]. 2011 Mar [cited 2019 Jan 20];27(3):510-20. Available from: http:// dx.doi.org/10.1590/S0102-311X2011000300011

15. Rasmussen AR, Wohlfahrt-Veje C, Renzy-Martin KT, Hagen CP, Tinggaard J, Mouritsen A, et al. Validity of self-assessment of pubertal maturation [Internet]. Pediatrics [Internet]. 2015 Jan [cited 2019 Feb 16]. Available from: https://doi.org/10.1542/peds.2014-0793

16. World Health Organization - WHO. BMI-for-age (5-19 years) [Internet]. Geneva: World Health Organization; 2007 [cited 2018 Nov 21]. Available from: http://www. who.int/growthref/who2007_bmi_for_age/en/

17. Kakeshita IS, Silva AIP, Zanatta DP, Almeida SS. Construção e fidedignidade teste reteste de escalas de silhuetas brasileiras para adultos e crianças. Psicol Teor e Pesqui [Internet]. 2009 abr-jun [citado 2020 set 9];25(2):263-70. Disponível em: http:// dx.doi.org/10.1590/S0102-37722009000200015

18. Gama SR. Excesso de peso em crianças moradoras em uma favela do Rio de Janeiro: uma abordagem sistêmica [Internet]. Rio de Janeiro: Escola Nacional de Saúde Pública Sérgio Arouca - ENSP/Fiocruz; 2016 [citado 2020 set 9]. 117 p. Disponível em: http://bvssp. icict.fiocruz.br/lildbi/docsonline/pdf/gamasrd.pdf

19. Sichieri R. Epidemiologia da obesidade. Rio de Janeiro: Ed. UERJ; 1998. 140 p.

20. R Core Team. R: a language and environment for statistical computing [Internet]. [S.1.]: R Foundation for Statistical Computing; 2016 [cited 2018 Apr 8]. Available from: http://www.r-project.org/

21. Manios Y, Moschonis G, Karatzi K, Androutsos 0, Chinapaw M, Moreno LA, et al. Large proportions 
of overweight and obese children, as well as their parents, underestimate children's weight status across Europe. The ENERGY (EuropeaN Energy balance Research to prevent excessive weight Gain among Youth) project. Public Health Nutr [Internet]. 2015 Aug [cited 2020 Sep 9];18(12):2183-90. Available from: https://doi.org/10.1017/s136898001400305x

22. Thompson AL, Adair L, Bentley ME. "Whatever average is": understanding african american mothers' perceptions of infant weight, growth, and health. Curr Anthropol [Internet]. 2014 Jun [cited 2020 Sep 9];55(3):348-55. Available from: https://doi.org/10.1086/676476

23. Nery M. The new middle class in Brazil: the bright side of the poor [Internet]. Rio de Janeiro: FGV/CPS; 2010 [cited 2020 Sep 9]. 134 p. Available from: https://www. cps.fgv.br/ibrecps/ncm2010_eng/NMC_Research_ FGV_CPS_Neri_FORMAT_ENG_FIM_apres_SITE.pdf

24. Gama SR, Cardoso L0, Rubinsztajn IK, Fischer A, Carvalho MS. Feeding children in a favela in Rio de Janeiro, Brazil: how much is spent and what would be the cost of a healthy diet? Rev Bras Saúde Materno Infant [Internet]. 2015 Oct-Dec [cited 2019 Jan 13];15(4):425-34. Available from: https:// doi.org/10.1590/S1519-38292015000400006

25. Monteiro CA, Benicio MHD, Konno SC, Silva ACF, Lima ALL, et al. Causas do declínio da desnutrição infantil no Brasil, 1996-2007. Rev Saúde Pública [Internet]. 2009 fev [citado 2020 jan 12];43(1):35-43. Disponível em: https://doi.org/10.1590/S0034-89102009000100005

26. Cunningham SA, Chandrasekar EK, Cartwright K, Yount KM. Protecting children's health in a calorie-surplus context: household structure and child growth in the United States. PLoS One [Internet]. 2019 Aug [cited 2019 0ct 13];14(8):e0220802. Available from: https://doi.org/10.1371/journal.pone.0220802

27. Diniz E, Koller SH. Fatores associados à gravidez em adolescentes brasileiros de baixa renda. Paidéia (Ribeirão Preto) [Internet]. 2012 set- dez [citado 2019 out 13];22(53):305-14. Disponível em: https:// doi.org/10.1590/S0103-863X2012000300002

28. Batista Filho M, Melo MNT. Food and toys. Cad Saúde Pública [Internet]. 2013 Jan [cited 2020 Sep 9];29(1):10-2. Available from: https://doi. org/10.1590/s0102-311x2013000100003

29. Cai L, Zhang T, Ma J, Ma L, Jing J, Chen Y. Self-perception of weight status and its association with weight-related knowledge, attitudes, and behaviors among Chinese children in Guangzhou. J Epidemiol [Internet]. 2017 Jul [cited 2020 Jan 20];27(7):338-45. Available from: https://doi.org/10.1016/j.je.2016.08.011

30. Sweet E. Symbolic capital, consumption, and health inequality. Am J Public Health [Internet]. 2011 Feb [cite 2019 Apr 10];101(2):260-4. Available from: https://dx.doi.org/10.2105\%2FAJPH.2010.193896 


\section{Abstract}

Objective: To analyze agreement/discrepancy between body self-image and Body Mass Index (BMI), according to variables related to type of food and environment influence. Methods: This was a cross-sectional analysis of 195 prepubescent children ( $\geq 5$ years), attending a Primary Health Care service in Manguinhos, Rio de Janeiro. Z-scores were applied to classify BMI. Self-image was collected using the figure rating scale (silhouettes) validated for children. A multinomial model was used to estimate covariate association with the underestimated/ overestimated BMI outcome in relation to self-image. Results: Overweight children underestimated their BMI, as compared with self-image, more often (58.6\%) than obese children (22.0\%) and children with adequate BMI (49.0\%). This dissociation was correlated to participation in a cash transfer program (OR=2.01 - 95\%CI 1.04;3.90) and daily consumption of sugar-sweetened foodstuffs (OR=3.88 - 95\%CI 1.05;14.39). Conclusion: Underestimation of BMI among overweight children should be taken into account by Primary Health Care services, in order to enhance intervention practices.

Keywords: Body Image; Childhood Obesity; Body Mass Index; Primary Health Care.

\section{Resumen}

objetivo: Verificar la correspondencia/divergencia entre autoimagen e indice de masa corporal (IMC), según variables relacionadas al tipo de alimentación e influencia del ambiente. Métodos: Análisis transversal de 195 niños prepúberes ( $\geq 5$ años), atendidos en la Atención Primaria de Salud (APS). Se utilizó la clasificación del IMC basada em la puntuación z de la Organización Mundial de la Salud. La autoimagen se evaluó según la escala de silueta validada para niños. Se estimó la asociación de covariables con el resultado de la subestimación/sobreestimación del estado nutricional en modelo multinomial. Resultados: Los niños con sobrepeso subestimaron su IMC, en comparación con la autoimagen, en una mayor proporción (58.6\%) que los obesos (22.0\%) y eutróficos (49.0\%). Esta disociación de la representación se asoció con la participación en el programa de transferencia de renta (odds ratio [OR] $\left.=2,01-I C_{95 \%} 1,04 ; 3,90\right)$ y con el consumo diario de dulces (OR=3,88-IC $C_{95 \%}$ 1,05;14,39). Conclusión: La subestimación del IMC de los niños con sobrepeso debe tenerse en cuenta para la mejor intervención en las prácticas de atención primaria de salud.

Palabras clave: Imagen Corporal; Obesidad Pediátrica; Índice de Masa Corporal; Atención Primaria de Salud; Estudios Transversales.

Recebido em 19/03/2020

Aprovado em 18/08/2020 